\title{
Association of phosphodiesterase 4D gene and interleukin-6 receptor gene polymorphisms with ischemic stroke in a Chinese hypertensive population
}

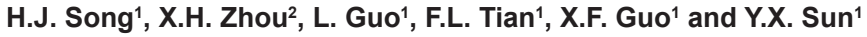 \\ 1 Department of Cardiovascular Medicine, \\ The First Affiliated Hospital of China Medical University, Shenyang, Liaoning, China \\ ${ }^{2}$ Department of Cardiovascular Medicine, \\ The First Affiliated Hospital of Lanzhou Medical College, Lanzhou, Gansu, China \\ Corresponding author: Y.X. Sun \\ E-mail: songhongjj@163.com
}

Genet. Mol. Res. 14 (4): 19396-19403 (2015)

Received August 13, 2015

Accepted October 2, 2015

Published December 29, 2015

DOI http://dx.doi.org/10.4238/2015.December.29.50

ABSTRACT. Genetic factors have been shown to be associated with the risk of stroke. However, due to individual differences, the extent of the association between genetic factors and stroke varies widely. Hypertension is considered one of the most important risk factors for stroke, but it remains unknown whether the genetic association with stroke in a hypertensive population is the same as that in a non-hypertensive population. The aim of the present study was to explore the association between the phosphodiesterase 4D gene (PDE4D) and interleukin-6 receptor gene (IL6R) single nucleotide polymorphisms and ischemic stroke in a hypertensive population. The study included 307 ischemic stroke cases with hypertension and 227 controls (simple hypertension). The polymorphic loci rs 12188950 and rs918592 in PDE4D, and rs4075015 and rs4537545 in IL6R were selected for analyzing the genotype and allele frequencies between cases and controls. rs 12188950 was not found in the 
study population. In the univariate analysis, the rs918592 polymorphism in PDE4D was found to be significantly associated with ischemic stroke with the recessive model $(P=0.02)$, whereas no association with ischemic stroke was observed for rs4075015 and rs4537545 in IL6R. Following adjustment for binary logistic regression, the rs918592 polymorphism was not found to be associated with ischemic stroke. While prior studies have found an association between PDE4D and IL6R polymorphisms and ischemic stroke, our results suggest that this association may be different in a hypertensive population. Therefore, the association between PDE4D and IL6R polymorphisms and ischemic stroke among a hypertensive population requires further investigation.

Key words: Stroke; PDE4D; IL6R; Hypertensive population

\section{INTRODUCTION}

Stroke is an important cause of death in the world. The high morbidity and mortality rates of stroke are a large burden to societies, and hence stroke is a global public health concern. There are numerous risk factors that are associated with stroke, of which genetic factors play a particularly important role in the pathogenesis of stroke (Graffagnino et al., 1994; Qureshi et al., 2004). The development of molecular biology techniques and completion of the Human Genome Project have led to case-control association studies using single nucleotide polymorphism (SNP) as markers for disease (Rosand and Altshuler, 2003). Correlation analyses are useful in identifying stroke susceptibility genes, and provide insight into the pathogenesis of stroke. Multiple-genetic polymorphisms that are associated with stroke have been identified (Mitchell et al., 2014). However, results are varied due to differences in ethnicity, number of study cases, and the diagnostic criteria for stroke (Nilsson-Ardnor et al., 2005; Saleheen et al., 2005).

The gene encoding phosphodiesterase 4D (PDE4D) is considered to be closely associated with stroke. This association was first reported in Iceland in 2003 (Gretarsdottir et al., 2003). The gene is located on human autonomic $5 q 12$, and the protein product of PDE4D is a cyclic nucleoside phosphodiesterase (PDE4D), which is widely distributed in the human body, particularly in inflammatory cells (Gretarsdottir et al., 2003). PDE4D degrades second-messenger cyclic adenosine monophosphate (CAMP), which may reduce neointimal damage and prevent smooth muscle cell proliferation (Jones and Hall, 2004). Low levels of cAMP caused by the biological effects of PDE4D can lead to the proliferation and migration of vascular smooth muscle cells, which are processes involved in atherosclerosis (Le Jeune et al., 2002).

Interleukin-6 (IL-6) is a multifunctional cytokine that plays an important role in numerous diseases. The biological effects IL-6 are elicited through its specific receptor, IL-6R. It has been shown that IL-6R induces acute phase protein synthesis during inflammation and trauma (Mackiewicz et al., 1992). IL-6 gene polymorphisms are closely associated with type 2 diabetes (Wang et al., 2005), acute myocardial infarction (Zawawi et al., 1995), and ischemic stroke (Jung et al., 2011). However, the association between IL-6R and ischemic stroke has not been investigated.

While these previous findings have important implications, they require replication in other populations, as half of strokes are accompanied by hypertension. The aim of the present study was to evaluate $P D E 4 D$ and the IL-6R gene (IL6R) as susceptibility genes for ischemic stroke in 
a population of stroke patients with hypertension and matched controls from northern China using haplotype association tests.

\section{MATERIAL and METHODS}

\section{Study population}

The present study was conducted in Fuxin, Liaoning, China. A baseline survey was conducted between 2004 and 2006 to assess a total of 45,925 selected cases, in which the response rate was $84.8 \%$. All groups were followed up once every 2 years. The primary study was a prospective, population-based epidemiological study from a register that consecutively included any patients with hypertension and first-ever stroke from Fuxin. A total of 307 (174 males and 133 females; mean age, 61.86 years) first-ever ischemic stroke patients with hypertension, included in the Stroke Register between March 1, 2004 and September 30, 2010, participated in the study. Two hundred and twenty-seven control subjects were also included (124 males and 103 females; mean age, 63.35 years), without stroke and from the same geographical area, randomly selected from the same population with similar age, gender distribution, and other characteristics. Information concerning vascular risk factors, including hypertension, heart disease, diabetes, and smoking was collected from patients and control subjects using a questionnaire. Blood samples for DNA extraction were obtained from ischemic stroke and control patients who had agreed (personally or through their next of kin) to participate in the study. The research protocol was approved by China Medical University Research Ethics Committee and written informed consent was obtained from all patients or their guardians.

\section{Blood sample preparation}

DNA extraction reagents were provided by the Shanghai General Biological Engineering Co., Ltd. (Shanghai, China). DNA was extracted according to manufacturer instructions. DNA samples were then stored at $-20^{\circ} \mathrm{C}$ until the analysis. All samples were subject to stringent quality control.

\section{Definition of ischemic stroke and hypertension}

Blood pressure (BP) was measured three times and the use of antihypertensive drugs was assessed by a standard questionnaire. Hypertension was defined as average systolic BP $\geq 140 \mathrm{mmHg}$, and/or average diastolic BP $\geq 90 \mathrm{mmHg}$, and/or use of antihypertensive medication within 2 weeks of the questionnaire. The diagnosis of stroke was made according to the World Health Organization criteria. All patients in the study underwent computed tomography or magnetic resonance scans, or had autopsy findings compatible with ischemic stroke.

\section{Smoking habit definition}

Smoking habit was defined by current smoking status. Current smokers were individuals who smoked $\geq 1$ tobacco products daily (Teo et al., 2006) in the previous 12 months, including those who had discontinued smoking within the past year. 


\section{Genotyping}

Genotyping was performed by multiplex SNaPshot technology using an ABI fluorescencebased assay discrimination method (Applied Biosystems, Foster City, CA, USA), which has been described in detail in previous studies (Di Cristofaro et al., 2010; Hu et al., 2012). The rs12188950 and rs918592 polymorphisms in PDE4D and rs4075015 and rs4537545 in IL6R were identified by polymerase chain reaction as previously described (Fernandez-Real et al., 2000). Detection of single-base extended probe primers was based on fluorescence and extended length, which was detected by capillary electrophoresis on the ABI3730XL Sequencer (Applied Biosystems).

\section{Statistical analysis}

Deviations of genotype distributions from Hardy-Weinberg equilibrium in this haplotypebased case-control study were evaluated using the SHEsis software (http://analysis.bio-X.cn) (Shi and $\mathrm{He}, 2005)$. Continuous variables are reported as means $\pm \mathrm{SD}$, and categorical variables are reported as percentages. Differences between the means of the variables of the case and control groups were evaluated by two-sample Student $t$-tests for continuous variables, and $\chi^{2}$ tests for categorical variables were performed to evaluate differences in genotype distribution. Odds ratios (ORs) and $95 \%$ confidence intervals $(95 \% \mathrm{Cls})$ were calculated to assess the association between PDE4D polymorphisms and ischemic stroke. $\mathrm{P}$ values $<0.05$ were considered to be statistically significant. All tests were performed using two-tailed hypotheses. Data analyses were completed using the SPSS 17.0 software (SPSS Inc., Chicago, IL, USA).

\section{RESULTS}

A total of 307 cases of stroke with hypertension and 227 controls were included in the present study. Demographic and personal characteristics of the stroke patients and control subjects are presented in Table 1. There were no significant differences in demographic characteristics between the two groups, with the exception of high-density lipoprotein. The mean age of the case group was $\sim 1.5$ years younger than that of the control group, whereas the proportion of people $\geq 60$ years of age was also lower in the case group compared to that of the control group.

Table 1. Characteristics of cases and controls.
\begin{tabular}{lcrc}
\hline & Stroke patients $(\mathrm{N}=307)$ & Control subjects $(\mathrm{N}=227)$ & $\mathrm{P}$ value \\
\hline Male (\%) & $174(56.7 \%)$ & $124(54.6 \%)$ & 0.655 \\
Age (years) & $61.86 \pm 8.77$ & $63.35 \pm 7.92$ & 0.055 \\
Mean arterial pressure (mmHg) & $121.49 \pm 14.64$ & $119.11 \pm 13.53$ & 0.062 \\
Smoke (\%) & $138(45.0 \%)$ & $109(48.1 \%)$ & 0.317 \\
High-density lipoprotein level (mM) & $1.43 \pm 0.29$ & $1.48 \pm 0.36$ & 0.055 \\
Low-density lipoprotein (mM) & $2.99 \pm 0.71$ & $2.91 \pm 0.77$ & 0.169 \\
BMl (kg/m²) & $23.77 \pm 3.26$ & $23.53 \pm 4.31$ & 0.475 \\
Fasting glucose (mM) & $5.98 \pm 1.99$ & $6.15 \pm 2.33$ & 0.363 \\
\hline
\end{tabular}

$\mathrm{BMI}=$ body mass index. Values are reported as means $\pm \mathrm{SD}$ or $\%$.

Single-marker association tests were performed for rs12188950 and rs918592 in PDE4D and rs4075015 and rs4537545 in IL6R. The SNaP shot analysis did not reveal any variation in rs12188950 between cases and controls. The allele frequencies for the other three SNPs of 
PDE4D and IL6R are shown in Table 2. The allele frequency of rs918592 was higher in the case group compared to that in the control group for both genders (males, 75.9 vs $69.2 \%$; females, 77.4 vs $72.8 \%$ ). For rs 4075015 , the T-allele frequency was higher in the case group compared to that in the control group in males (81 vs $77.5 \%)$, but was approximately the same in females (85.7 vs $85.4 \%$ ). The C-allele frequency in rs 4537545 was lower in the case group compared to that in the control group for both genders (males, 83.3 vs $85.8 \%$; females, 72.9 vs $75.7 \%$ ) (Table 2).

\begin{tabular}{|c|c|c|c|c|c|}
\hline \multirow[t]{2}{*}{ Individuals (No. of cases/controls) } & \multirow[t]{2}{*}{ SNP } & \multirow[t]{2}{*}{ Allele } & \multicolumn{2}{|c|}{ Frequency $(\%)$} & \multirow[t]{2}{*}{ MAF } \\
\hline & & & Case & Control & \\
\hline \multirow[t]{4}{*}{ Male $(174 / 124)$} & rs918592 & A & 75.9 & 69.2 & 0.416 \\
\hline & rs12188950 & $\mathrm{T}$ & 100.0 & 100.0 & 0.092 \\
\hline & rs 4075015 & $\mathrm{~T}$ & 81.0 & 77.5 & 0.371 \\
\hline & rs4537545 & $\mathrm{C}$ & 83.3 & 85.8 & 0.46 \\
\hline \multirow[t]{4}{*}{ Female $(133 / 103)$} & rs918592 & G & 77.4 & 72.8 & 0.416 \\
\hline & rs12188950 & $\mathrm{T}$ & 100.0 & 100.0 & 0.092 \\
\hline & rs 4075015 & $\mathrm{~T}$ & 85.7 & 85.4 & 0.371 \\
\hline & rs4537545 & $\mathrm{C}$ & 72.9 & 75.7 & 0.46 \\
\hline
\end{tabular}

SNP = single nucleotide polymorphisms; MAF = minor allele frequency.

As polymorphisms were not found for rs12188950, it was excluded from subsequent experiments. The genotype distribution of PDE4D and IL6R SNPs of the groups is shown in Table 3. Results of the univariate analysis revealed that the rs918592 polymorphism was significantly associated with ischemic stroke in the recessive model $(P=0.02)$, but not in the additive or dominant models (additive, $\mathrm{P}=0.061$; dominant, $\mathrm{P}=0.116$ ). However, rs4075015 and rs4537545 in IL6R were not associated with ischemic stroke in any of the three models. Therefore, the rs918592 polymorphism in PDE4D was selected for subsequent experiments.

\begin{tabular}{|c|c|c|c|c|c|}
\hline Genotype & Case $[(\mathrm{N}=307) \mathrm{N}(\%)]$ & Control $[(\mathrm{N}=227) \mathrm{N}(\%)]$ & Additive $P$ value & Dominant $P$ value & Recessive P value \\
\hline \multicolumn{6}{|l|}{ rs918592 } \\
\hline $\mathrm{AA}$ & $72(23.5)$ & $65(28.6)$ & 0.061 & 0.116 & $0.020^{*}$ \\
\hline$A G$ & $149(48.5)$ & $100(44.1)$ & & & \\
\hline GG & $86(28.0)$ & $62(27.3)$ & & & \\
\hline \multicolumn{6}{|l|}{ rs4075015 } \\
\hline AA & $52(16.9)$ & $42(18.5)$ & 0.805 & 0.553 & 0.639 \\
\hline AT & $146(47.6)$ & $110(48.5)$ & & & \\
\hline TT & $109(35.5)$ & 75 (33.0) & & & \\
\hline \multicolumn{6}{|l|}{ rs 4537545} \\
\hline $\mathrm{CC}$ & 97 (31.6) & $70(30.8)$ & 0.836 & 0.852 & 0.549 \\
\hline $\mathrm{CT}$ & $158(51.5)$ & $114(50.2)$ & & & \\
\hline TT & $52(16.9)$ & $43(18.9)$ & & & \\
\hline
\end{tabular}

${ }^{*} \mathrm{P}<0.05$ indicates statistical significance.

As shown in Table 4, following adjustment for binary logistic regression, the rs918592 polymorphism was found to be not associated with ischemic stroke in any of the three models (adjusted $\mathrm{OR}=0.874,95 \% \mathrm{Cl}=0.686-1.115$ and $\mathrm{P}=0.279$ in the additive model; OR $=0.906$, $95 \% \mathrm{Cl}=0.609-1.348$ and $\mathrm{P}=0.627$ in the dominant model; $\mathrm{OR}=1.31,95 \% \mathrm{Cl}=0.875-1.961$ and $\mathrm{P}=0.004$ in the recessive model). 
Table 4. Associated between rs918592 variation and ischemic stroke in additive, dominant, and recessive models after adjustment for binary logistic regression.

\begin{tabular}{lcccc}
\hline & Wald & P value & OR & $95 \% \mathrm{Cl}$ of OR \\
\hline Additive & 1.170 & 0.279 & 0.874 & $0.686-1.115$ \\
Dominant & 2.360 & 0.627 & 0.906 & $0.609-1.348$ \\
Recessive & 1.718 & 0.190 & 1.312 & $0.875-1.961$ \\
\hline
\end{tabular}

$\mathrm{P}<0.05$ indicates statistical significance.

\section{DISCUSSION}

In the present study, two SNPs (rs12188950 and rs918592) in PDE4D, which were found to be associated with stroke in the DECODE study in an Icelandic population (Helgadottir et al., 2004), and two SNPs (rs4075015 and rs4537545) in IL6R were investigated for association with ischemic stroke. The results demonstrated that the PDE4D and IL6R polymorphisms were not associated with ischemic stroke in a Chinese hypertensive population.

$P D E 4 D$, and other members of the phosphodiesterase family, are expressed in the majority of inflammatory cells where the cAMP-dependent and -independent members regulate immune responses (Tilley and Maurice, 2005). The cAMP pathway affects the recruitment of neutrophils by modifying selective chemotaxis and adhesion, by which the neutrophils then participate in arterial inflammation that influences ischemic stroke (Grond-Ginsbach et al., 2008).

In 2003, the DECODE genetics research group in Iceland conducted a genome-wide screening of stroke susceptibility genes. The results showed that PDE4D was most closely associated with stroke with a particularly significant correlation between PDE $4 D$ and carotid endogenous and cardioembolic stroke (Gretarsdottir et al., 2002; Song et al., 2006). However, there are relatively limited studies on this association. A study by Liao et al. (2010) demonstrated that there was a significant association between rs12188950 and ischemic stroke, but no such correlation was found in a large sample population study conducted in Sweden (Lovkvist et al., 2012). The rs12188950 polymorphism in PDE4D has been shown to be a protective factor for ischemic stroke in other populations (Lovkvist et al., 2008). A recent meta-analysis showed a significant association between PDE4D and ischemic stroke (Yoon et al., 2011). However, an exact correlation between PDE4D polymorphisms and ischemic stroke remains controversial.

The present study revealed no association between the rs12188950 SNP and ischemic stroke. This observation may be due to genetic differences between ethnicities in different study populations. The results showed that no differences in the rs12188950 genotype were detected, which was found to be T/T. Hence, the rs12188950 polymorphism did not exist in the current study population. A study of Caucasians and African-Americans in the US also has shown that there was no polymorphism of rs12188950 (Lovkvist et al., 2012). Recently, a study on hospitalized Chinese patients was conducted, and the authors concluded that the rs918592 polymorphism was significantly associated with ischemic stroke (Song et al., 2012). However, the results of the present study are not in accordance with those results.

IL-6 is a pleiotropic cytokine that is secreted by various types of cells, including lymphocytes, endothelial cells, fibroblasts, muscle cells, and adipocytes. IL-6 has a variety of important physiological functions and is involved in glucose and lipid metabolism. The biological effects of IL-6 are exerted through the IL-6R in cells that express of IL6R. The IL-6 gene has been found to be associated with cardiovascular diseases (Schieffer et al., 2000; Luc et al., 2003; 
Anderson et al., 2013). Peripheral blood and cerebrospinal fluid of stroke patients contains high levels of IL-6, which causes an overexpression of IL-6R in cells that are sensitive to IL-6 (Bauer et al., 1989). However, no such association was found in the present study.

Ischemic stroke is a complex disease caused by the interaction of genetic and environmental factors. Genetic factors can affect the incubation period of vascular occlusion infarct size, or lead directly to the formation of the ischemic stroke (Dudbridge, 2003; Dichgans, 2007). However, the identification of susceptibility genes of ischemic stroke is an extremely complex process. The association between gene polymorphisms and ischemic stroke may be influenced by geographical factors and ethnicity. Despite this, a number of prior studies have found an association between $P D E 4 D$ and IL6R polymorphisms and ischemic stroke. Hypertension is a major risk factor for stroke. Evidence has shown that stroke patients with or without hypertension exhibit different clinical features and pathophysiology (Arboix et al., 2004). The results of the present study were consistent with these findings.

The present study also had certain limitations. First, the study was a case-control study that included subjects only from Liaoning in China. The results may have been different if subjects were included from different ethnicities and geographic regions. Second, the degree of atherosclerosis was not quantified in the current study. Third, the study was based on a small sample size, and hence the results should be confirmed in a larger study. Therefore, additional studies are required to evaluate the association between $P D E 4 D$ and IL6R polymorphisms and the risk of ischemic stroke among a hypertensive population.

\section{Conflicts of interest}

The authors declare no conflict of interest.

\section{ACKNOWLEDGMENTS}

The authors would like to thank Dr. Yundi Jiao and Dr. Xinghu Zhou for the assistance and support provided during the present study.

\section{REFERENCES}

Anderson DR, Poterucha JT, Mikuls TR, Duryee MJ, et al. (2013). IL-6 and its receptors in coronary artery disease and acute myocardial infarction. Cytokine 62: 395-400.

Arboix A, Roig H, Rossich R, Martinez EM, et al. (2004). Differences between hypertensive and non-hypertensive ischemic stroke. Eur. J. Neurol. 11: 687-692.

Bauer J, Bauer TM, Kalb T, Taga T, et al. (1989). Regulation of interleukin 6 receptor expression in human monocytes and monocyte-derived macrophages. Comparison with the expression in human hepatocytes. J. Exp. Med. 170: 1537-1549.

Di Cristofaro J, Silvy M, Chiaroni J and Bailly P (2010). Single PCR multiplex SNaP shot reaction for detection of eleven blood group nucleotide polymorphisms: optimization, validation, and one year of routine clinical use. J. Mol. Diagn. 12: 453-460. Dichgans M (2007). Genetics of ischaemic stroke. Lancet Neurol. 6: 149-161.

Dudbridge F (2003). Pedigree disequilibrium tests for multilocus haplotypes. Genet. Epidemiol. 25: 115-121.

Fernandez-Real JM, Broch M, Vendrell J, Richart C, et al. (2000). Interleukin-6 gene polymorphism and lipid abnormalities in healthy subjects. J. Clin. Endocrinol. Metab. 85: 1334-1339.

Graffagnino C, Gasecki AP, Doig GS and Hachinski VC (1994). The importance of family history in cerebrovascular disease. Stroke 25: 1599-1604.

Gretarsdottir S, Sveinbjornsdottir S, Jonsson HH, Jakobsson F, et al. (2002). Localization of a susceptibility gene for common forms of stroke to 5q12. Am. J. Human Genet. 70: 593-603. 
Gretarsdottir S, Thorleifsson G, Reynisdottir ST, Manolescu A, et al. (2003). The gene encoding phosphodiesterase 4D confers risk of ischemic stroke. Nat. Genet. 35: 131-138.

Grond-Ginsbach C, Hummel M, Wiest T, Horstmann S, et al. (2008). Gene expression in human peripheral blood mononuclear cells upon acute ischemic stroke. J. Neurol. 255: 723-731.

Helgadottir A, Manolescu A, Thorleifsson G, Gretarsdottir S, et al. (2004). The gene encoding 5-lipoxygenase activating protein confers risk of myocardial infarction and stroke. Nat. Genet. 36: 233-239.

Hu XP, Wu JQ, Zhu LP, Wang X, et al. (2012). Association of Fcgamma receptor IIB polymorphism with cryptococcal meningitis in HIV-uninfected Chinese patients. PLoS One 7: e42439.

Jones DW and Hall JE (2004). Seventh report of the Joint National Committee on Prevention, Detection, Evaluation, and Treatment of High Blood Pressure and evidence from new hypertension trials. Hypertension 43: 1-3.

Jung JE, Kim GS and Chan PH (2011). Neuroprotection by interleukin- 6 is mediated by signal transducer and activator of transcription 3 and antioxidative signaling in ischemic stroke. Stroke 42: 3574-3579.

Le Jeune IR, Shepherd M, Van Heeke G, Houslay MD, et al. (2002). Cyclic AMP-dependent transcriptional up-regulation of phosphodiesterase 4D5 in human airway smooth muscle cells. Identification and characterization of a novel PDE4D5 promoter. J. Biol. Chem. 277: 35980-35989.

Liao YC, Lin HF, Guo YC, Yu ML, et al. (2010). Sex-differential genetic effect of phosphodiesterase 4D (PDE4D) on carotid atherosclerosis. BMC Med. Genet. 11: 93.

Lovkvist H, Smith JG, Luthman H, Hoglund P, et al. (2008). Ischaemic stroke in hypertensive patients is associated with variations in the PDE4D genome region. Eur. J. Human Genet. 16: 1117-1125.

Lovkvist H, Olsson S, Hoglund P, Melander O, et al. (2012). A large-sample assessment of possible association between ischaemic stroke and rs12188950 in the PDE4D gene. Eur. J. Human Genet. 20: 783-789.

Luc G, Bard JM, Juhan-Vague I, Ferrieres J, et al. (2003). C-reactive protein, interleukin-6, and fibrinogen as predictors of coronary heart disease: the PRIME study. Arterioscler. Thromb. Vasc. Biol. 23: 1255-1261.

Mackiewicz A, Schooltink H, Heinrich PC and Rose-John S (1992). Complex of soluble human IL-6-receptor/IL-6 up-regulates expression of acute-phase proteins. J. Immunol. 149: 2021-2027.

Mitchell BD, Fornage M, McArdle PF, Cheng YC, et al. (2014). Using previously genotyped controls in genome-wide association studies (GWAS): application to the Stroke Genetics Network (SiGN). Front. Genet. 5: 95.

Nilsson-Ardnor S, Wiklund PG, Lindgren P, Nilsson AK, et al. (2005). Linkage of ischemic stroke to the PDE4D region on 5q in a Swedish population. Stroke 36: 1666-1671.

Qureshi AI, Suri MF, Kirmani JF and Divani AA (2004). The relative impact of inadequate primary and secondary prevention on cardiovascular mortality in the United States. Stroke 35: 2346-2350.

Rosand J and Altshuler D (2003). Human genome sequence variation and the search for genes influencing stroke. Stroke 34 : 2512-2516.

Saleheen D, Bukhari S, Haider SR, Nazir A, et al. (2005). Association of phosphodiesterase 4D gene with ischemic stroke in a Pakistani population. Stroke 36: 2275-2277.

Schieffer B, Schieffer E, Hilfiker-Kleiner D, Hilfiker A, et al. (2000). Expression of angiotensin II and interleukin 6 in human coronary atherosclerotic plaques: potential implications for inflammation and plaque instability. Circulation 101: 1372-1378.

Shi YY and He L (2005). SHEsis, a powerful software platform for analyses of linkage disequilibrium, haplotype construction, and genetic association at polymorphism loci. Cell Res. 15: 97-98.

Song C, Zhou X, Dong Q, Fan R, et al. (2012). Regulation of inflammatory response in human chondrocytes by lentiviral mediated RNA interference against S100A10. Inflamm. Res. 61: 1219-1227.

Song Q, Cole JW, O'Connell JR, Stine OC, et al. (2006). Phosphodiesterase 4D polymorphisms and the risk of cerebral infarction in a biracial population: the Stroke Prevention in Young Women Study. Human Mol. Genet. 15: $2468-2478$.

Teo KK, Ounpuu S, Hawken S, Pandey MR, et al. (2006). Tobacco use and risk of myocardial infarction in 52 countries in the INTERHEART study: a case-control study. Lancet 368: 647-658.

Tilley DG and Maurice DH (2005). Vascular smooth muscle cell phenotype-dependent phosphodiesterase 4D short form expression: role of differential histone acetylation on cAMP-regulated function. Mol. Pharmacol. 68: 596-605.

Wang H, Zhang Z, Chu W, Hale T, et al. (2005). Molecular screening and association analyses of the interleukin 6 receptor gene variants with type 2 diabetes, diabetic nephropathy, and insulin sensitivity. J. Clin. Endocrinol. Metab. 90: 1123-1129.

Yoon D, Park SK, Kang D, Park T, et al. (2011). Meta-analysis of homogeneous subgroups reveals association between PDE4D gene variants and ischemic stroke. Neuroepidemiology 36: 213-222.

Zawawi TH, Alyafi WA, Hashim IA, Kholeif MA, et al. (1995). The value of serum interleukin-6 measurement in the investigation of patients suspected of myocardial infarction. Acta Cardiol. 50: 387-396. 